This information is current as of April 25, 2023. 


\title{
Percutaneous CT-Guided Biopsies of the Cervical Spine: Technique, Histopathologic and Microbiologic Yield, and Safety at a Single Academic Institution
}

\author{
(D)E.L. Wiesner, (D)T.J. Hillen, (D). Long, and (DJ.W. Jennings
}

\begin{abstract}
BACKGROUND AND PURPOSE: Cervical spine biopsies can be challenging due to the anatomy and the adjacent critical structures. Percutaneous image-guided biopsies can obviate the need for an open biopsy, however there have been few studies looking at the approaches, safety, and efficacy of percutaneous cervical spine biopsies. This retrospective study evaluated technical considerations, histopathologic and microbiologic yield, and safety in CT-guided cervical bone biopsies.
\end{abstract}

MATERIALS AND METHODS: A retrospective review of cervical bone and/or bone/disc biopsies performed from January 2010 to January 2017 was included in this study. Clinical diagnosis and indication, patient demographics, biopsy location, biopsy needle type, technical approach, lesion size, dose-length product, conscious sedation details, complications, and diagnostic histopathologic and/or microbiologic yield were recorded for each case and summarized.

RESULTS: A total of 73 patients underwent CT-guided cervical bone biopsies. Fifty-three percent (39/73) were for clinical/imaging concern for infection and $47 \%$ (34/73) were for primary tumors or metastatic disease. Thirty-four percent (25/73) were of the inferior cervical spine (ie, C6 and C7). A sufficient sample was obtained for histopathologic and microbiologic analyses in 96\% (70/73) of the biopsies. Forty-six percent (18/39) of those samples taken for infection had positive cultures. Two intraprocedural complications occurred in which the patients became hypotensive during the procedure without long-term complications.

CONCLUSIONS: Percutaneous CT-guided biopsy of the cervical spine is an effective and safe procedure with high diagnostic yield and can obviate open procedures for histopathologic and microbiologic analyses of patients with clinical and imaging findings concerning for infection or primary and metastatic osseous lesions.

ABBREVIATION: DLP $=$ dose-length product

B iopsies of the cervical spine are more difficult to obtain than those of the thoracic or lumbar regions due to the anatomy and smaller size of the vertebral elements as well as the critical adjacent vascular and neural anatomy.

Although the demands for cervical spine biopsies are steadily increasing in our practice, few publications address the safety of such biopsies and the high diagnostic yield they can provide. To date, the largest study was published in 2016 by Cox et $\mathrm{al}^{1}$ and looked at CT-guided cervical bone biopsies in 43 patients by combining data from 2 large tertiary care hospitals. A diagnosis was obtained in 41 of the 43 patients, for a yield of $95 \%$. In 2008 , Rimondi et $\mathrm{al}^{2}$ published

Received December 8, 2017; accepted after revision January 24, 2018.

From the Mallinckrodt Institute of Radiology, Washington University School of Medicine, St. Louis, Missouri.

Please address correspondence to Elizabeth L. Wiesner, BA, Mallinckrodt Institute of Radiology, 510 South Kingshighway Blvd, St. Louis, MO 63110; e-mail: wiesner.elizabeth@wustl.edu

http://dx.doi.org/10.3174/ajnr.A5603 a study on 430 CT-guided spinal biopsies, 10 of which were within the cervical spine $(2.3 \%$ of the sample). The diagnostic accuracy for this study was $70 \%$. Similarly, in 2004, Lis et $\mathrm{al}^{3}$ studied the accuracy of CT-guided spinal biopsies. There were 410 cases in all, with only 9 cases being of the cervical spine. Yet, for these 9 cases, there was a $100 \%$ diagnostic accuracy reported.

Using a minimally invasive percutaneous CT-guided approach to cervical spine biopsies can obviate open biopsies for both infection and primary or metastatic lesions. This technique lends itself to the increasing demands for cultures by infectious disease specialists and for tissue diagnosis of metastatic disease by oncologists.

The purpose of this retrospective study was to further evaluate technical considerations, histopathologic and microbiologic yield, and safety in CT-guided cervical bone biopsies.

\section{MATERIALS AND METHODS}

Patient Selection and Study Enrollment

Approval from the institutional review board was granted with a waiver of patient informed consent. Correlating data from a single 
academic institution on cervical bone and/or bone/disc biopsies from January 2010 to January 2017 were then gathered and included in this study. For each case, we recorded and summarized the following: patient demographics, clinical diagnosis and indication, level biopsied, biopsy needle type/gauge, technical approach, number and length of each soft-tissue/bone core biopsy, CT dose-length product, conscious sedation details including sedation time and the amount of fentanyl and/or midazolam (Versed) given, and diagnostic histopathologic and/or microbiologic yield. Procedural complications were documented according to the Society of Interventional Radiology classification. ${ }^{4} \mathrm{~Pa}-$ tients were clinically monitored for at least 1 hour after each procedure for evidence of acute complications such as hematoma formation or neurologic injury. Electronic medical records were also reviewed for evidence of delayed complications within 30 days of the biopsy.

Table 1: Biopsy needles used for 34 bone lesion biopsies ${ }^{a}$

\begin{tabular}{lc}
\hline \multicolumn{1}{c}{ Biopsy Needle } & Bone Lesion Samples \\
\hline Bonopty 14/15 (AprioMed) & 8 \\
Arrow OnControl 11/13 (Teleflex) & 12 \\
Arrow OnControl 12/14 (Teleflex) & 5 \\
Achieve 16 (CareFusion) & 5 \\
Bard 14 (Bard Peripheral Vascular) & 5 \\
Bard 16 (Bard Peripheral Vascular) & 1 \\
Tru-Cut 18 (CareFusion) & 3 \\
Tru-Cut 20 (CareFusion) & 1
\end{tabular}

${ }^{\text {a }}$ Six procedures used both bone and soft-tissue biopsy needle systems for 40 total needle systems used.

Table 2: Biopsy needles used for 39 infection biopsies ${ }^{a}$

\begin{tabular}{lc}
\hline \multicolumn{1}{c}{ Biopsy Needle } & Soft-Tissue Samples \\
\hline Bonopty 14/15 (AprioMed) & 16 \\
Arrow OnControl 11/13 (Teleflex) & 20 \\
Arrow OnControl 12/14 (Teleflex) & 3 \\
Achieve 14 (CareFusion) & 1 \\
Achieve 16 (CareFusion) & 1 \\
Achieve 18 (CareFusion) & 1 \\
Temno Evolution 18 (CareFusion) & 2 \\
Tru-Cut 16 (CareFusion) & 1 \\
Tru-Cut 20 (CareFusion) & 1
\end{tabular}

a Seven procedures used both bone and soft-tissue biopsy needle systems, 9 procedures used only soft-tissue needle systems, and 23 procedures used only bone biopsy needle systems for 46 total needle systems used.
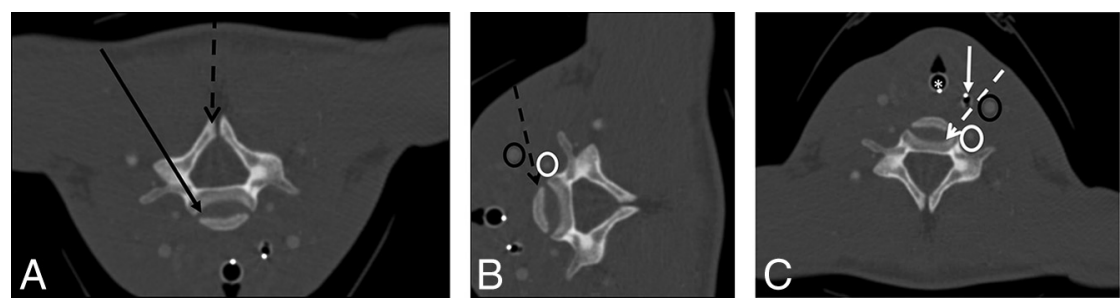

FIG 1. CT-guided cervical spine biopsies were performed using anterolateral, posterolateral, posterior, or lateral approaches. A, CT angiogram of the neck flipped vertically to depict prone positioning for a posterolateral- or posterior-approach cervical spine biopsy. The black arrow demonstrates a posterolateral-approach biopsy of the vertebral body or disc. The dashed black arrow demonstrates a directly posterior approach to a lesion in the spinous process. $B, C T$ angiogram of the neck rotated to depict decubitus positioning for a lateral-approach biopsy to the vertebral body (dashed black arrow). Note that the course of the biopsy needle is between the carotid artery (black oval) and vertebral artery (white oval). C, CT angiogram of the neck with the patient in a supine position for an anterolateral-approach cervical spine biopsy. The needle (dashed white arrow) passes between the intubated trachea (white asterisk), nasogastric tube/esophagus (solid white arrow), carotid artery (black oval), and vertebral artery (white oval).

\section{Biopsy Procedure}

procedures were performed by 1 of 6 board-certified fellowrequired. In most cases, a 1:1 mixture of bupivacaine $0.25 \%$ and lidocaine $1 \%$ was administered for subcutaneous and periosteal anesthesia. Average sedation time was 67.5 minutes; average intravenous fentanyl and Versed dosages were $210.1 \mu \mathrm{g}$ and $3.7 \mathrm{mg}$, respectively.

The Arrow OnControl Powered Bone Lesion Biopsy System (AprioMed, Lonnderry, New Hampshire) bone biopsy system were used for one access and biopsy of the sites of infection, sclerotic lesions, or Arrow OnControl 11/13 needle system, used in 32 of 64 procedures. Of the 73 total biopsy procedures performed, 22 required the use of a soft-tissue biopsy needle. Nine of the 73 biopsies were performed with only a soft-tissue coaxial needle system. The most commonly used soft-tissue biopsy needle was the Achieve 16-ga biopsy needle. It was used in 6 of the 22 procedures in which a soft-tissue biopsy needle was used. Tables 1 and 2 describe in detail the different biopsy needles used for infection and lesion biopsy.

Patient positioning on the table was dependent on the location of the cervical spine lesion and the approach used to perform the biopsy. For the posterolateral and directly posterior approaches, the patient was placed prone on the table. For the directly lateral approach, the patient was placed in the decubitus position with the side of the lesion up. For the anterolateral approach, the patient was placed supine. Posterolateral (60 procedures), directly posterior ( 1 procedure), directly lateral ( 3 procedures), or anterolateral (9 procedures) approaches were used to sample the lesions (Fig 1), depending on the location of the pathology. Our most commonly used approach was a posterolateral approach through the lateral mass into the vertebral body. This approach allows avoidance of important neurovascular structures in the neck but is tedious to perform because the needle has the farthest to travel within bone to get to the site of abnormality. A direct posterior approach was used for a single spinous process lesion. The anterolateral approach was used occasionally. This approach is somewhat treacherous because the needle must be threaded among multiple important structures, includ- 
ing the carotid artery laterally and the trachea and esophagus medially, and it is often difficult to avoid the thyroid for lesions in the lower cervical spine. We commonly used this approach if the patient had a prior radical neck dissection because there were fewer important structures to avoid in these cases. A lateral approach was used in cases in which the mass extended into the lateral soft-tissue or was near midline at a site deemed too difficult to reach by a posterolateral approach. In this approach, the needle needs to be passed between the external and internal carotid arteries and the vertebral artery. The internal jugular vein can sometimes be avoided but not always with this approach. With this approach in the upper cervical spine, it is imperative that the course of the vertebral artery is known because the location is variable.

CT angiography was occasionally performed immediately before the biopsy to better delineate the vascular anatomy and plan the safest biopsy path to minimize the risk of vascular injury. Of the 73 patients who underwent biopsy, 6 had corresponding CT angiograms. In these cases, an average of $80.0 \mathrm{~mL}$ of ioversol contrast (Optiray 350; Mallinckrodt, St. Louis, Missouri) was given intravenously to help localize vascular structures in relation to the tumor (Figs 2 and 3).

All samples were analyzed at the institution where the biopsy was performed.
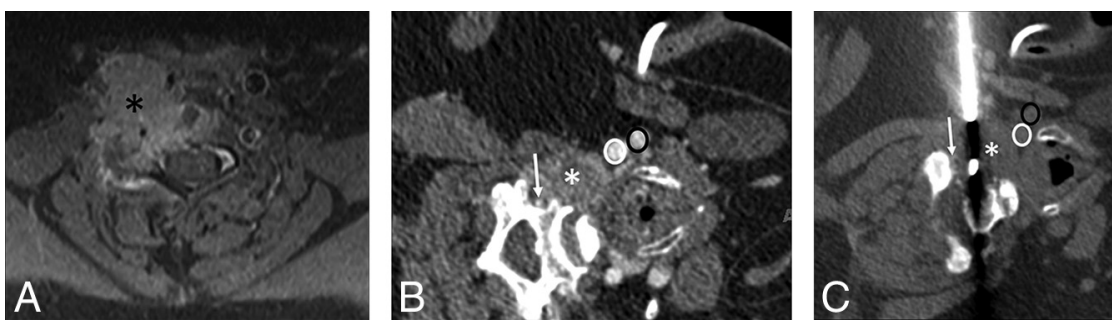

FIG 2. A 70-year-old woman with a history of lung cancer and a new right $C 5$ mass extending into the adjacent soft tissues. $A$, Axial $T 1$ fat-suppressed postcontrast MR image with a large right $C 5$ mass (black asterisk) extending into the adjacent soft tissues and vertebral canal. B, Intraprocedural CT angiogram with the patient in a left lateral decubitus position shows the mass (white asterisk) and adjacent vascular structures (vertebral artery, white arrow; common carotid artery, white oval; jugular vein, black oval). C, Intraprocedural CT image with the patient in the left lateral decubitus position demonstrates the biopsy using a coaxial soft-tissue biopsy needle passing between vascular structures (vertebral artery, white arrow; common carotid artery, white oval; jugular vein, black oval) into the mass (white asterisk) using a lateral approach. The surgical pathology result was metastatic lung adenocarcinoma.
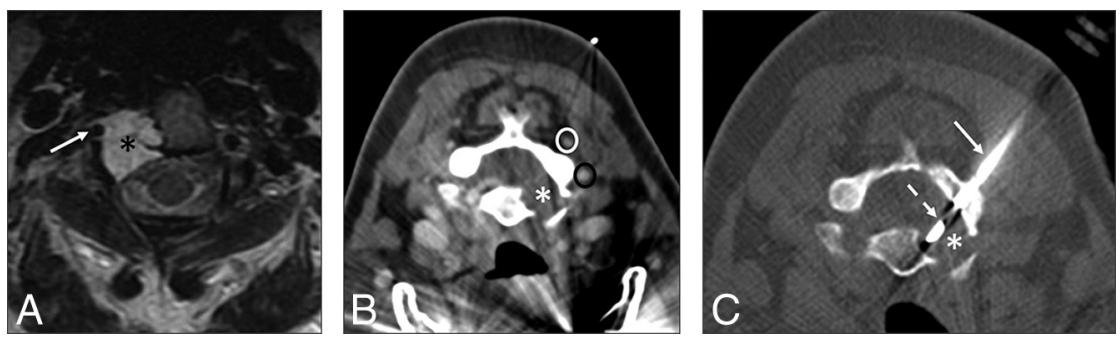

FIG 3. A 55-year-old woman with right neck and arm pain. A, Axial T2-weighted MR image with a large right $\mathrm{C} 2$ vertebral mass (black asterisk) surrounding the vertebral artery (white arrow). $B$, Intraprocedural CT angiogram soft-tissue-windowed image with the patient prone shows the mass (white asterisk) and adjacent vascular structures (deep cervical vein, white oval; vertebral artery, black oval). C, Intraprocedural CT bone-windowed image with the patient prone demonstrates the bone-access needle (white arrow) in the posterolateral right lamina of C2 and a soft-tissue biopsy needle (dashed white arrow) placed coaxially into the mass (white asterisk), using a posterolateral approach. The surgical pathology result was chordoma.

\section{RESULTS}

\section{Patient Characteristics}

A total of 73 patients underwent CTguided cervical bone biopsies. Sixty-two percent of the patients were men and $38 \%$ were women. Fifty-three percent (39/73) of the cervical biopsies were for clinical/imaging concern for infection (Fig 4), and 47\% (34/73) were for primary tumors or metastatic disease. A sufficient sample was obtained for histopathologic and microbiologic analyses in $96 \%(70 / 73)$ of the biopsies. Forty-six percent (18/39) of those samples taken for infection had positive culture growth from a sample. Two intraprocedural complications occurred. One patient became hypotensive and did not respond to the IV bolus; the procedure was terminated after 2 samples had already been obtained. The patient subsequently stabilized and had no further complications. The second patient became hypotensive and bradycardic before the biopsy needle could be placed. The patient subsequently stabilized and had no further complications. A cervical spine biopsy was performed 2 days later without complication.

\section{Lesion Characteristics}

Of the 34 CT-guided cervical bone biopsies that were for primary tumors or
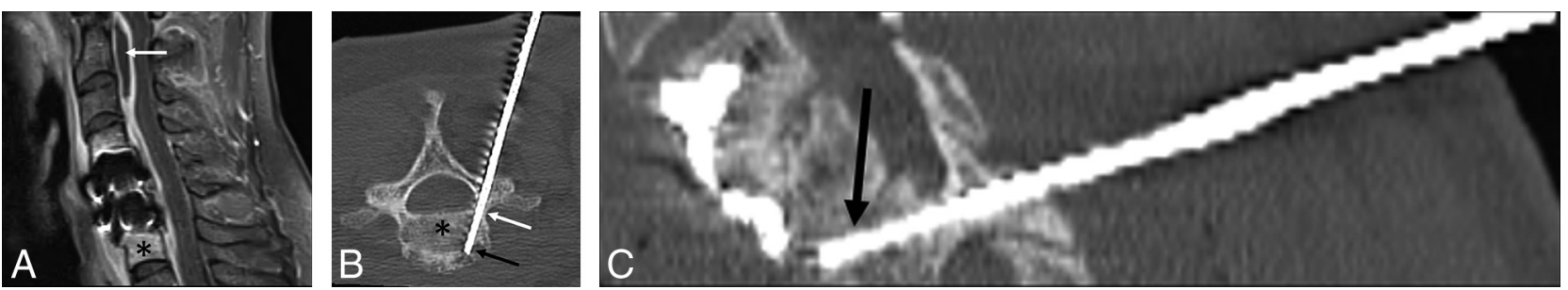

FIG 4. A 51-year-old woman with prior discectomy and interbody fusion. A, Sagittal Tl fat-suppressed postcontrast MR image with evidence of osteomyelitis at C7 (black asterisk), prevertebral inflammation, and epidural abscess (white arrow). Intraprocedural oblique axial (B) and oblique sagittal (C) CT reconstructed images during the biopsy procedure show the biopsy needle placed from a posterolateral approach, through the right C7 pedicle (white arrow) into the vertebral body (black asterisk) and subsequently the C6-C7 disc space (black arrow). Microbiology grew Staphylococcus aureus, and pathology showed osteomyelitis. 
Table 3: Histopathologic results of CT-guided cervical bone biopsies for primary tumors or metastatic disease

\begin{tabular}{ll}
\hline Malignant ( $n=24$ cases) & 4 \\
Myeloma & 4 \\
NSCLC/adenocarcinoma & 3 \\
Osteoid osteoma & 2 \\
Chordoma & 2 \\
Giant cell tumor & 2 \\
Indeterminate (abnormal) & \\
Metastatic carcinoma & 2 \\
Unknown primary & 1 \\
Epithelioid hemangioendothelioma & 1 \\
Metastatic carcinoma & \\
Breast primary & 1 \\
Metastatic papillary & 1 \\
Thyroid carcinoma & 1 \\
Aneurysmal bone cyst focal & \\
Eosinophilic granuloma & \\
Benign (n = 10 cases) & 6 \\
Normal & \\
Marrow fibrosis & 1 \\
Chronic inflammation & 1 \\
Micrococcus species & 1 \\
Osteomyelitis & 1 \\
\hline Nerems & 1
\end{tabular}

Note:-NSCLC indicates non-small-cell lung carcinoma.

${ }^{a}$ Normal means biopsy demonstrating normal bone seen on histopathology.

metastatic disease, the type of lesions biopsied included lytic, sclerotic, mixed, or CT occult. These lesions were classified as follows: 70.6\% (24/34) lytic, 17.6\% (6/34) mixed, 5.9\% (2/34) CT occult, and $5.9 \%(2 / 34)$ sclerotic. Histopathologic yield per lesion type was the following: $95.8 \%$ (23/24) lytic, $83.3 \%$ (5/6) mixed, $0.0 \%$ (0/2) CT occult, and 50.0\% (1/2) sclerotic.

To obtain an average size, we measured the longest axis of each lesion with an average lesion size of $18.2 \mathrm{~mm}$, ranging between 5.0 and $40.0 \mathrm{~mm}$. Ten lesions involved the entire vertebral body. Of the 34 biopsies performed for bone lesions in our study, the most common location of lesions, including the 10 cases in which the lesion encompassed the entire vertebral body, was the $\mathrm{C} 7$ cervical vertebra, which involved $29.4 \%$ (10/34) of the lesions. At this level, 5 were within the vertebral body, 3 were in the transverse process, and 2 were in the lateral mass. The locations of the lesions that made up the remaining 24/34 biopsies were as follows: C1 lesions $(2 / 24)$, with both lesions in the vertebral body/anterior arch; C2 lesions (7/24), with 4 lesions centered in the vertebral body alone, 2 lesions centered within the lamina, and 1 centered in the lateral mass; C3 lesions (2/24), both of which included the entire vertebral body; C4 lesions (2/34), of which 1 lesion was centered within the vertebral body and 1 lesion was located within the C4 transverse process; C5 lesions (4/24), with 2 lesions centered within the vertebral body, 1 lesion within the C5 spinous process, and 1 lesion centered in the lateral mass; and C6 lesions (7/24), with 6 lesions centered in the vertebral body and 1 lesion centered within the lateral mass.

Of the 34 biopsies obtained, 70.6\% (24) were malignant. The most common malignant lesions were myeloma and adenocarcinoma, each found in 4 patients (Table 3 ).

\section{CT Radiation Dose}

Our retrospective study also looked at dose-length product (DLP) in all $73 \mathrm{CT}$-guided cervical bone biopsies. For the 34 biopsies that were specific to primary tumors or metastatic disease, the range for DLP was 122-1767 $\mathrm{mGy} \times \mathrm{cm}$, with an average of $712 \mathrm{mGy} \times$ $\mathrm{cm}$. For the 39 biopsies specific to clinical/imaging concern for infection, the range for DLP was $163-3223 \mathrm{mGy} \times \mathrm{cm}$, with an average of $932.4 \mathrm{mGy} \times \mathrm{cm}$. Combining DLPs for all 76 spinal biopsies yielded the same range of $122-3223 \mathrm{mGy} \times \mathrm{cm}$, with an overall average DLP of $829.8 \mathrm{mGy} \times \mathrm{cm}$. We accounted for the range of DLPs by equating outliers with those patients who received ablation on the same day that the cervical biopsy was performed, therefore increasing the patient's DLP and thus our calculated DLP average.

When considering the most recent spinal biopsies, specifically from January 2015 to January 2017, which accounts for $47.9 \%$ (35/73) of all biopsies performed, there was a decreased average DLP of $639 \mathrm{mGy} \times \mathrm{cm}$. This difference in average DLPs within biopsies of the last 2 years can be associated with the increasing awareness of scanning parameters, advanced scanner technology, as well as institutional emphasis on decreased diagnostic and procedural radiation doses.

\section{DISCUSSION}

Multiple publications have demonstrated the safety and utility of percutaneous bone biopsies with fewer focusing on the spine and even fewer on the cervical spine. To our knowledge, this is the largest study to date on percutaneous CT-guided cervical bone biopsies. Compared with similar studies of spinal bone biopsies, our diagnostic yield is within the average, around 96\% for histopathologic and microbiologic analyses of all biopsies.

The high diagnostic yield of not just cervical bone biopsies but also biopsies throughout the entire spine is important to consider when looking at past imaging techniques and the nonspecific nature of radiologic imaging alone for yielding a definitive diagnosis. In 2012, a study by Kaltsikis et $\mathrm{al}^{5}$ on core needle biopsies of spinal lesions similarly emphasized the importance of the CTguided bone biopsies themselves to render more definitive histopathologic diagnoses. For their study, 79 biopsies of the spine were performed, 5 of the cervical spine, with a diagnostic yield of 95\% and a diagnostic accuracy of 97.3\%. Most spinal biopsies (52\%) performed in the study by Kaltsikis et al also followed our technique using the posterior lateral approach during biopsy.

Given the relatively smaller spinal anatomy and the ascending importance of the neural anatomy of the cervical spine, choosing the appropriate anatomic approach to safely perform the biopsy is an important consideration. We most commonly use a posterolateral approach for biopsy of the cervical spine, but other approaches to consider include the lateral, anterolateral, and directly posterior approach, depending on the location of the lesion. Of the 73 patients who underwent the procedure at our institution, there were only 2 minor intraprocedural complications and no postprocedural complications. There was no vascular or neurologic injury.

In 2013, Huang et $\mathrm{al}^{6}$ published a 2-year prospective study on the incidence of complications following percutaneous CTguided biopsies of spinal lesions, including soft tissues and bone. Of the 386 patients, the reported rates of bruising, level of discomfort/pain, and fever did not exceed $16.1 \%$ in any category. In 2006, $\mathrm{Peh}^{7}$ demonstrated low complication rates on CT-guided biop- 
sies of the spine, ranging from $0 \%$ to $10 \%$, with $<1 \%$ risk of major complication.

\section{CONCLUSIONS}

This study supports the addition of percutaneous cervical spine biopsies to the growing number of studies demonstrating the safety and efficacy of spine biopsies that can obviate open biopsies and their associated risks.

Disclosures: Travis J. Hillen—UNRELATED: Consultancy: Medtronic, Merit Medical Systems, Comments: consultant (Medtronic) and proctor training labs (Merit Medical Systems). Jack W. Jennings—UNRELATED: Consultancy: Merit, Medtronic, Bard, Comments: consultant (Merit, Medtronic, Bard) and advisory board (Merit, Medtronic).

\section{REFERENCES}

1. Cox M, Pukenas B, Poplawski M, et al. CT-guided cervical bone biopsy in $\mathbf{4 3}$ patients: diagnostic yield and safety at two large tertiary care hospitals. Acad Radiol 2016;23:1372-75 CrossRef Medline
2. Rimondi E, Staals E, Errani C, et al. Percutaneous CT-guided biopsy of the spine: results of 430 biopsies. Eur Spine J 2008;17:975-81 CrossRef Medline

3. Lis E, Bilsky M, Pisinski L, et al. Percutaneous CT-guided biopsy of osseous lesion of the spine in patients with known or suspected malignancy. AJNR Am J Neuroradiol 2004;25:1583-88 Medline

4. Omary R, Bettmann M, Cardella J, et al; Society of Interventional Radiology Standards of Practice Committee. Quality improvement guidelines for the reporting and archiving of interventional radiology procedures. J Vasc Interv Radiol 2003;14:S293-95 CrossRef Medline

5. Kaltsikis I, Chourmouzi D, Drevelegas K, et al. Core needle biopsy of spinal lesions under CT guidance: review of 79 cases. J Neurol Surg A Cent Eur Neurosurg 2012;73:199-203 CrossRef Medline

6. Huang A, Halpern E, Rosenthal DI. Incidence of delayed complications following percutaneous CT-guided biopsy of bone and soft tissue lesions of the spine and extremities: a 2-year prospective study and analysis of risk factors. Skeletal Radiol 2013;42:61-68 CrossRef Medline

7. Peh W. CT-guided percutaneous biopsy of spinal lesions. Biomed Imaging Interv J 2006;2:e25 Medline 